\title{
Study of iodine nutrition level in pregnant south Indian subjects in third trimester
}

\author{
Suresh Pichandi ${ }^{1}$, Desigamani Kanniyappan,, ${ }^{2}$ Janakiraman P $^{3}$, Ramadevi $K^{4}$ \\ ${ }^{1}$ Assistant Professor, Dept. of Biochemistry, Velammal Medical College Hospital \& RI, Madurai, Tamil Nadu, \\ ${ }^{2}$ Associate Professor, Dept. of Biochemistry, Tagore Medical College Hospital, Chennai, Tamil Nadu, ${ }^{3}$ Tutor, \\ Dept. of Community Medicine, PES Institute of Medical Sciences and Research, Kupam, Andra Pradesh, India, \\ ${ }^{4}$ Director, Professor \& HOD, Dept. of Biochemistry, Madras Medical College, Chennai, Tamil Nadu, India
}

*Corresponding Author:

Email: desigamani@ tagoremch.com

\begin{abstract}
Objective: Aim of the present study is to analyse the nutritional status of iodine among pregnant women by measuring urinary excretion and do the correlation with thyroid hormone and auto antibodies.

Materials and Methods: one hundred pregnant women with third trimester gestation and one hundred agematched non-pregnant were included in study.

Results: Urinary Iodine excretion and serum TSH, fT4, fT3, AMA and ATG were estimated were estimated for case and control groups. The mean urinary iodine excretion concentration (UIC) in pregnant women was $290.17 \pm 91.71 \mu \mathrm{g} / \mathrm{L}$. Excess urinary iodine excretion was found in 69\%. There were elevated serum AMA levels in 36 pregnant women and that positive correlated with excess iodine.

Conclusion: The pregnant women had no iodine deficiency, rather had high urinary iodine concentrations indicating more than adequate iodine intake. The excess urinary iodine level may tiger the thyroid auto antibody.

Keywords: Urinary iodine excretion (UIE), thyroid stimulating hormone (TSH), free thyroxine (fT4), free triiodothyronine (fT3), anti-microsomal antibody (AMA), anti-thyroglobulin antibody (ATA), pregnancy, iodine excess.
\end{abstract}

Received: $07^{\text {th }}$ August, 2017

\section{Introduction}

Iodine is a micronutrient that is essential for the production of thyroid hormones and required for the normal functioning of various organs including thyroid and mammary gland. Adequate maternal iodine is important for normal fetal and infant neurogical growth. ${ }^{1}$ The major part of dietary iodine (>90\%) is excreted in the urine. ${ }^{2} \mathrm{~A}$ non-pregnant women urinary iodine exertion represents equilibrium of dietary intake, thyroidal iodine extraction, the total body thyroid hormone pool, and GFR. ${ }^{3}$

Dietary iodine requirements are increased in pregnancy and lactation due increased renal blood flow and glomerular filtration, which lead to increased iodine clearance and iodine loss in urine. The recommended daily intake of pregnancy-specific urinary iodine ranges are according to the World Health Organization, a urinary iodine excretion value below $150 \mu \mathrm{g} / \mathrm{L}$ is an indicator of iodine deficiency; values above $250 \mu \mathrm{g} / \mathrm{L}$ are considered to be above requirements and values above $500 \mu \mathrm{g} / \mathrm{L}$ indicate excessive in pregnancy. ${ }^{4}$ However, in pregnancy, the iodine intake is considered to be adequate when the urinary iodine concentration is between $150-250 \mu \mathrm{g} / \mathrm{L}$.
Accepted: $15^{\text {th }}$ December, 2017

The most serious adverse effect of iodine deficiency in pregnant and lactating women is damage to their fetuses, newborns and weaning infants. ${ }^{5}$ In contrast to iodine deficiency, excess exposure to iodine during pregnancy may cause fetal and neonatal thyroid dysfunction. However, the safe upper limit for chronic iodine ingestion in pregnancy and lactation is not currently well defined.

The Universal salt iodination program introduced for eliminates the iodine deficiency; the World Health Organization has recommended the measurement of urinary iodine as the standard method to assess the dietary intake of iodine by the community.

In India, after universal salt iodization, several reports has been published of normalization of iodine nutrition in school children as reflected by median urinary iodine (MUI) ${ }^{6-8}$ However, very few studies reported for iodine nutrition in pregnancy in north India ${ }^{9,10}$ and no specific study reports from South India. We have undertaken this study to assess iodine nutrition by urinary iodine excretion in pregnant women with third trimester subject attending a tertiary care centre in Chennai. 


\section{Materials and Methods}

A total of one hundred pregnant women in third trimester were included in this study. One hundred non-pregnant, age-matched, adult female were included as controls. This study sample were collected from the Madras medical college hospital and ESIC Medical College Hospital Chennai, Tamil Nadu, India.

All the pregnant women Thyroid hormones (TSH, fT4 and fT3 by ELISA method) and urinary iodine $^{11}$ were measured. The antibody titres - anti-microsomal antibodies (AMA) and anti-thyroglobulin antibodies (ATG) - (by ELISA method) were also measured in all pregnant women.

\section{Statistical Analysis}

The STATA13 version was used for data processing and analysis. Case and control quantitative data were presented as mean and standard deviation and comparison of means student t-test was used. Chi-square $(\chi 2)$ was used for categorical data. Kruskal-Wallis test were used with median and inter quarter range (IQR) to compare the nonparametric data among the group. Pearson's correlation was used to find associations between analytes.

\section{Results}

The mean age of the pregnant women was $23.88 \pm 4.24$ years, while that of the controls was $28.63 \pm 3.78$ years, with significant difference between the two groups $(\mathrm{P}=<0.001)$. The gestational age of pregnant women was $34.30 \pm 3.11$ weeks.

Mean urinary iodine concentration of pregnant women was $290.17 \pm 91.71 \mu \mathrm{g} / \mathrm{L}$ while that in the controls was $153.61 \pm 46.05 \mu \mathrm{g} / \mathrm{L}$. There was significant difference in the UIE between the pregnant women and controls ( $p$ value $<0.001$ ).

Based on the results obtained, the patients were classified into 2 types:

Type I: Urinary iodine excretion: Based on the urinary iodine level, the 100 pregnant women were classified into four groups [Fig. 2]. Using the World Health Organization (WHO) criteria, iodine nutrition, as assessed by the mean urinary iodine excretion of pregnant women, is described in Table 4. Only $4 \%$ of the pregnant women had iodine deficiency, while $69 \%$ had more than adequate iodine nutrition.

Type II: Physiological classification: Based on the thyroid hormone profile, and antibody titers, the 100 patients were classified into three groups as hypothyroid $(\mathrm{n}=15)$, hyperthyroid $(\mathrm{n}=5)$ and euthyroid (normal thyroid) subjects $(\mathrm{n}=80)$ [Fig. 1]. The anti-microsomal antibody value was positive in 36 pregnant subjects. (Fig. 3)

The comparison was done among hypothyroid, hyperthyroid and euthyroid pregnant women subject, median value of urinary iodine were 285(260-360), 360 (340$370)$ and 260(227.5-325) respectively and there were significant difference ( $\mathrm{p}$ value $<0.05$ ) in the median urinary iodine excretion across these groups (Table 3 \& Fig. 4).

Pearson's correlation was used to find the correlation and association between analysts. There was a significant correlation between UI and AMA ( $\mathrm{r}=0.5498, \mathrm{p}<0.01)$ in pregnant women subject. Also, a significant correlation between UI and ATG $(r=0.3192, p<0.01)$ was found in those subjects. (Table $4 \&$ Fig. 5, 6)

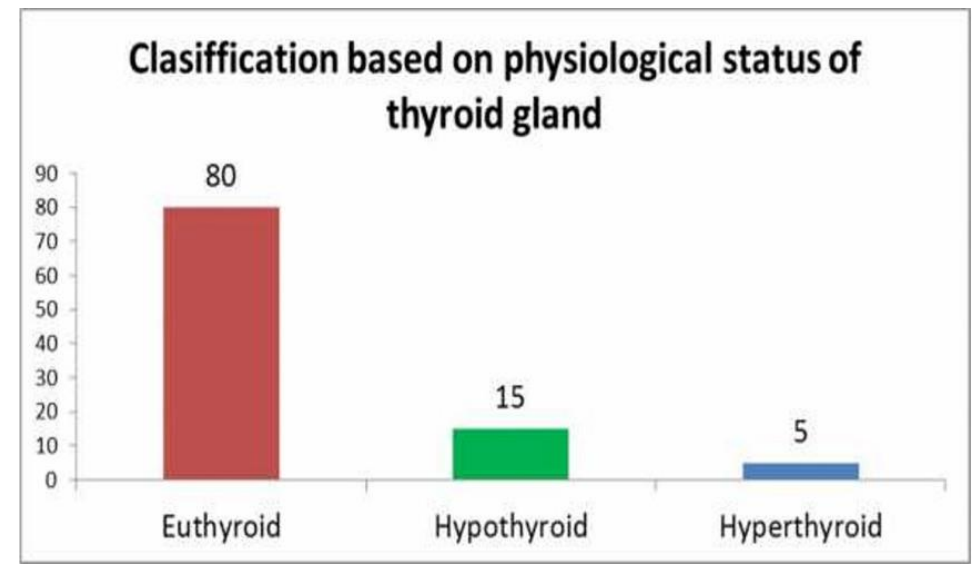

Fig. 1: Classification based on physiological status of thyroid gland 


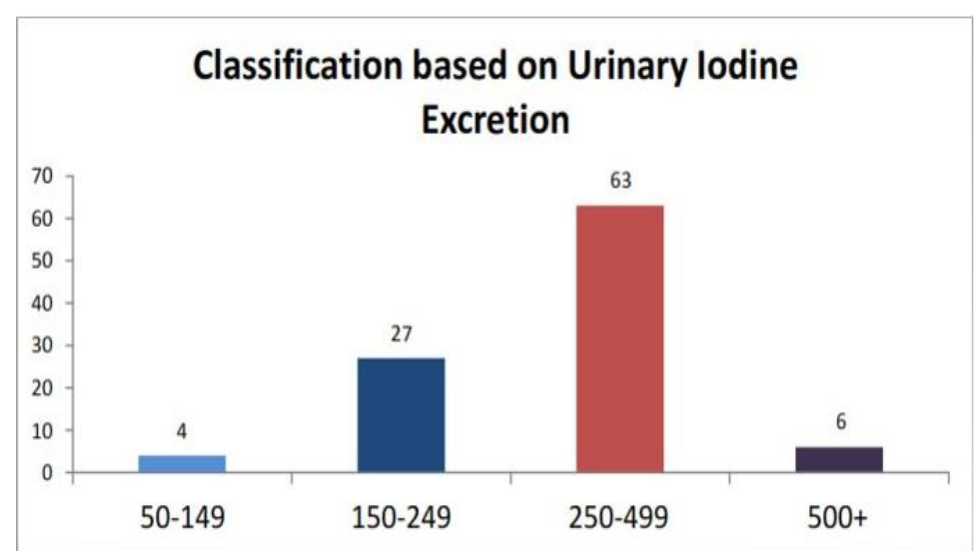

Fig. 2: Classification based on Urinary Iodine Excretion

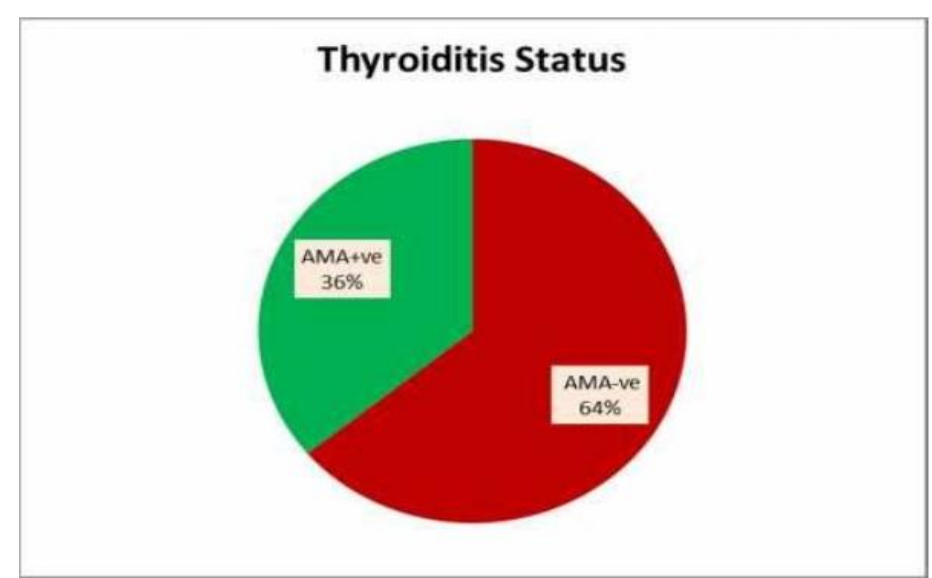

Fig. 3: Throiditis Status

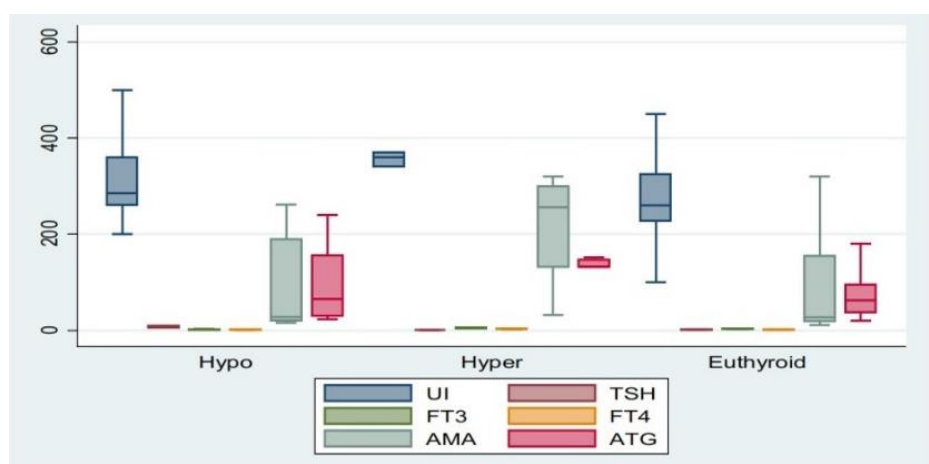

Data were presented as Median and inter quarter range (IQR). Data between three groups were compared using Kruskal-Wallis test

Fig. 4: Comprison of thyroid disorder based on physiological status of thyroid gland 


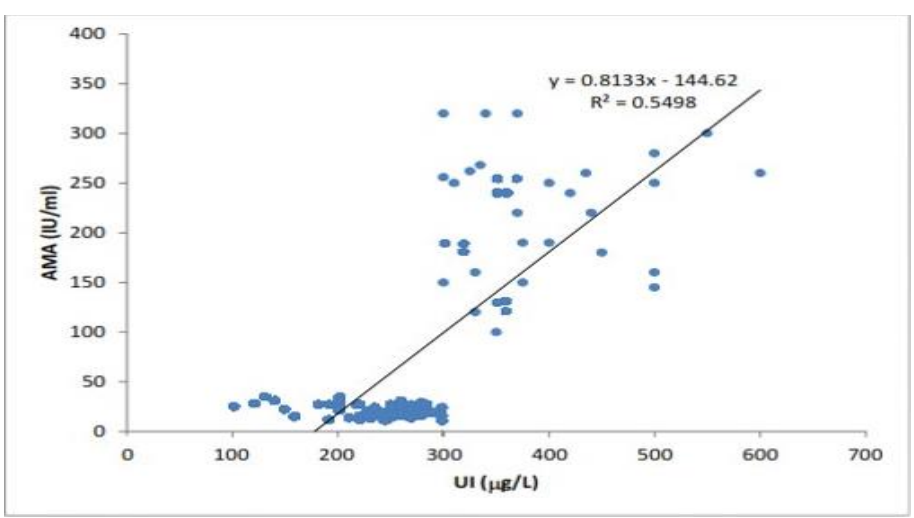

Fig. 5: UI vs. AMA

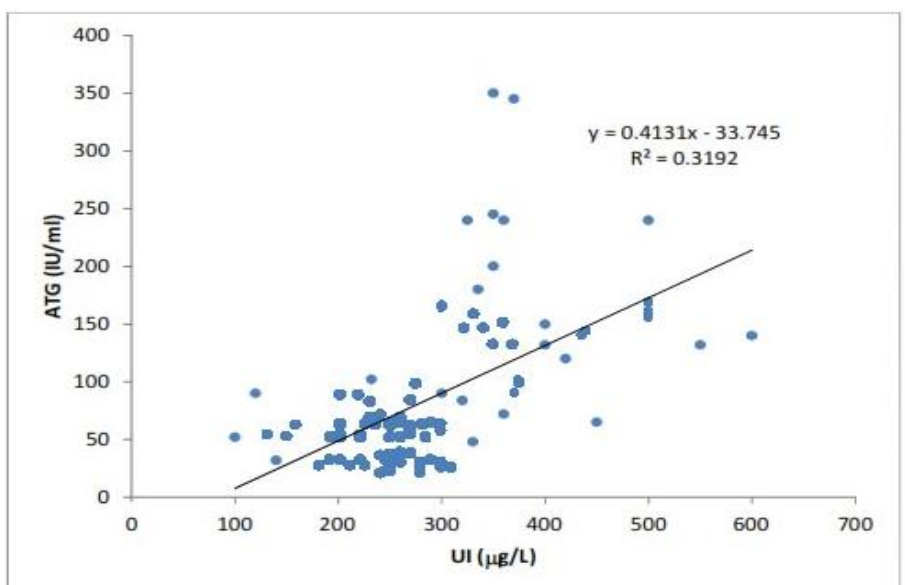

Fig. 6: UI vs. ATG

\section{Observation}

Iodine is an essential micronutrient throughout life. Various physiological changes increase iodine requirements during pregnancy because of Increase in renal iodine excretion, increase thyroid hormone production, iodine requirement for a fetus and iodine secretion into breast milk. ${ }^{12}$

In iodine-deficient areas, total body iodine stores decline gradually during pregnancy and results in impaired maternal and fetal thyroid hormone synthesis. ${ }^{13}$ Iodine deficiency has been endemic in India for a long time. Iodine sufficiency has been achieved in many parts of the country after Universal Salt Iodization (USI) in India.

In our study showed iodine excessed (above $250 \mu \mathrm{g} / \mathrm{L}$ ) in $69 \%$ of the subjects among that $6 \%$ had very high excretion of iodine $(>500 \mu \mathrm{g} / \mathrm{L})$, while $27 \%$ had normal iodine status (MUI 150$250 \mu \mathrm{g} / \mathrm{L})$. Only a small percentage (4\%) of the study group had iodine deficiency (less than 150 $\mu \mathrm{g} / \mathrm{L})$. The study group are classified based on thyroid hormone as hypothyroid $(\mathrm{n}=15)$, hyperthyroid $(n=5)$ and Euthyroid $(n=80)$ and there median value of urinary iodine was 285
(260-360), 360 (340-370) and 260(227.5-325). These reports indicating that excess urinary iodine excretion are hall mark for pregnant subject

Thyroiditis was observed in $36 \%$ of patients in this group. The excess iodine may be the reason for triggering autoimmunity in this study group. The correlation was done to assess the strength and relationships between the urinary iodine and thyroid hormones and auto-antibodies among pregnant women. The results of the present study showed good agreement among the different thyroid hormones with excess urinary iodine excretion. Urinary iodine was positively correlated with anti-microsomal antibody AMA $(\mathrm{r}=0.5498, \mathrm{p}<0.01)$, and anti-thyroglobulin antibody ATG $(r=0.3192, \mathrm{p}<0.01)$.The higher MUI in pregnant subjects may be due to an improvement in the implementation of USI, over time.

Limitations of our study is small hospital based study, Larger sample size communitybased studies are required in populations, to establish the reference ranges for urinary iodine concentration in pregnancy. Also in the hospital settings could not identify the Iodine status of the populations with respect to the dietary habits. 


\section{Conclusion}

The chances of iodine deficiency are much less in coastal areas like Tamil Nadu because of adequate amount of iodine is available in the coastal regions of our country. Universal salt iodination program has also eliminated the iodine deficiency as reported by many studies. Iodine excess is the hall mark that is observed among our patients in this study. The study comprised of 100 pregnant women with third trimester. The study group had a mean UI excretion of 290.17 $\mu \mathrm{g} / \mathrm{l}$. This shows majority of the pregnant women had increased urinary iodine excretion and suggested excess iodine intake. These group women had $36 \%$ AMA positive. Also, our study report showed positive correlation of urinary iodine with auto antibodies AMA and ATG. Excessive iodine intake may trigger thyroid autoimmunity

Risk-benefit analysis clearly is in favour of iodine supplementation, but at the same time speaks for a careful dosing, avoiding median intakes above the upper recommended level. This small hospital-based study for urinary iodine concentration in pregnant women may not be representative of community iodine nutrition and similar large number of sample required for community settings to find the iodine nutrition in pregnancy.

\section{Acknowledgements}

The authors thank all members have helped us enthusiastically in this work.

Funding: No funding sources

Conflict of Interest: None declared

\section{Reference}

1. Glinoer D. The regulation of thyroid function during normal pregnancy: Importance of the iodine nutrition status. Best Pract Res ClinEndocrinolMetab 2004;18:133-52.

2. Delange F. Optimal Iodine Nutrition during Pregnancy, Lactation and the Neonatal Period. Int J EndocrinolMetab 2004;2:1-12.

3. Davison JM, Dunlop W. Renal hemodynamics and tubular function in normal human pregnancy. Kidney Int 1980;18:152.

4. WHO, UNICEF, ICCIDD. Assessment of the iodine deficiency disorders and monitoring their elimination. A guide for programme managers. 3rd ed. Geneva: World Health Organization; 2007.

5. Hetzel BS. Iodine deficiency disorders (IDD) and their eradication. Lancet. 1983 Nov 12;2(8359):1126-9.

6. Marwaha RK, Tandon N, Gupta N, Karak AK, Verma K, Kochupillai N. Residual goitre in the postiodization phase: Iodine status, thiocyanate exposure and autoimmunity. ClinEndocrinol (Oxf) 2003;59:672-81.

7. Marwaha RK, Tandon N, Desai A, Kanwar R, Grewal K, Aggarwal R, et al. Reference range of thyroid hormones in normal Indian school-age children. ClinEndocrinol (Oxf) 2008;68:369-74.

8. Marwaha RK, Tandon N, Desai A, Kanwar R, Mani K. Iodine nutrition in upper socioeconomic school children of Delhi. Indian Pediatr 2010;47:335-8.

9. Smyth PP. Variation in iodine handling during normal pregnancy. Thyroid 1999;9:637-42.

10. Grewal E, Khadgawat R, Gupta. Assessment of iodine nutrition in pregnant north Indian subjects in three trimesters. Indian J EndocrMetab 2013;17(2);289-93.

11. Dunn JT, Crutchfield HE, Gutekunst R, Dunn AD. Two simple methods for measuring iodine in urine. Thyroid Off J Am Thyroid Assoc. 1993;3(2):119-23.

12. Burrow GN. Thyroid function and hyperfunction during gestation. Endocr Rev 1993;14:194-202.

13. Glinoer D (1997) The regulation of thyroid function in pregnancy: pathways of endocrine adaptation from physiology to pathology. Endocr Rev 18: 404-433. 